\title{
A New Back-Propagation Algorithm for Diagnosing Defective Casting
}

\author{
Nazri M. Nawi, Zawati Harun, and Noorhaniza Wahid
}

\begin{abstract}
The diagnosis of defective castings has always been a centre of attention in the manufacturing industry. An intelligent diagnosis system should be able to diagnose effectively the causal representation and also justify its diagnosis. Recently, the artificial-neural networks (ANN), or simply neural-networks (NN), technique has gained more popularity in learning cause and effect analysis in casting processes However, the algorithm comes with problems like slow convergence and convergence to local minima and unable to model exponential increase/decrease in belief values in cause and effect relationships. This paper proposed a new algorithm for improving the conventional back-propagation algorithm by introducing gain parameters during the learning process. The abilities of the proposed method to capture the exponential change in the belief variation of the cause when the belief in the effect is at its minimum is compared with the outputs from both the conventional back propagation on a real casting data set.
\end{abstract}

Index Terms-Back-propagation neural network, search direction, gain variation, optimization.

\section{INTRODUCTION}

Every day foundries manufacture a large number of castings. Every time a casting is produced, a large amount of data is generated involving process-parameter values and one or more indicators on whether the casting is defective or not. This data is encoded for each type of defect, for each day, week and month of the casting process and is available for all casting components.

The rejection data for a given casting and time frame, normally indicates a pattern, which has normally few defects occurring at significantly high proportions and some occurring at significantly low proportions. Therefore, the diagnostic casting problem was defined as recognising patterns in the casting rejection data and identifying a corresponding combination of causes. It was observed that a combination of defects generally occurs as a result of a combination of causes [1].

The cause and effect relationship in a casting process is complex and non-linear. Furthermore, a large number of parameters are needed to be coordinated with each other in an optimal way to minimise the occurrence of defective castings.

Manuscript received June 12, 2012; revised September 10, 2012. This work was supported in part by Ministry of Higher Education Malaysia under the Fundamental Research Grant Scheme (FRGS-737).

M. N. Nazri and N. Wahid are with the Faculty of Science Computer and Information Technology, Universiti Tun Hussein Onn Malaysia (UTHM), 86400, Parit Raja, Batu Pahat, Johor, MALAYSIA (e-mail: nazri@uthm.edu.my,nhaniza@uthm.edu.my).

Z. Harun is with the Faculty of Mechanical Engineering and Manufacturing, Universiti Tun Hussein Onn Malaysia (UTHM), 86400, Parit Raja, Batu Pahat, Johor, MALAYSIA (e-mail: zawati@uthm.edu.my).
This has led to the necessity of developing computer-based optimisation techniques. An optimisation process is a computational technique that determines an optimal value for process parameters such that the magnitude of one or more response variables of the process is minimised. It also ensures that the process operates within established limits or constraints [2]. Casting process optimisation has facilitated foundry men in making right choices, but it still remains a challenging area that has drawn the attention of many researchers during the last two decades.

Recent studies have used the response surface method (RSM) to optimise parameters in the casting process [3]. The computational efficiency of the RSM approach significantly reduces as the number of process parameters increase [4]. This is mainly because RSM techniques show the same limitations as showed by polynomial-regression techniques; the number of unknowns in the system increases exponentially with the number of parameters.

In contrast, Taguchi's robust design method provides a process engineer with a systematic and efficient approach for conducting experimentation to determine near optimum settings of design parameters for performance and cost [5]. The robust design method uses orthogonal arrays (OA) to study the parameter space, usually containing a large number of decision parameters, with a small number of experiments. To this date, a quite significant amount of research and development work has been done in order to optimise parameters of the casting process by using the Taguchi method [6].

Recently, the artificial-neural networks (ANN), or simply neural-networks ( $\mathrm{NN}$ ), technique has gained more popularity in learning cause and effect analysis in casting processes [7]. ANN consists of interconnected cells, called neurons, and simulates the behaviour of the biological neural network in a human brain [8]. Neural-networks' techniques are able to adapt, learn from examples and are generally used to model complex relationships between inputs and outputs or to classify data finding common patterns [9]. This ability makes the field of diagnosis a potential application for neural networks.

Back-propagation neural network (BPNN) provides very effective way in learning cause and effect analysis in casting processes [1]. However, the training process for a BPNN require the designers to arbitrarily select parameters such as network topology, initial weights and biases, learning rate value, the activation function, value for gain in activation function and momentum. An improper choice of any of these parameters can result in slow convergence or even network paralysis, where the training process comes to a standstill or get stuck at local minima. Many variations of this algorithm 
have been proposed by previous researchers to increase the BPNN training efficiency and one of the approaches is to adjust the slope of activation function [10].

$$
E=\frac{1}{2} \sum_{k}\left(t_{k}-o_{k}^{L}\right)^{2}
$$

This paper proposed a new algorithm by suggesting a simple modification to the search direction used by the current working back propagation algorithm and other almost all optimization method that has been summarized by Bishop [11]. The gradient based search direction is locally modified by a gain value used in the activation function of the corresponding node to improve the convergence rates respective of the optimization algorithm used.

$$
o_{j}^{s}=f\left(c_{j}^{s} n e t_{j}^{s}\right)
$$

The remaining of the paper is organized as follows: Section two illustrates the proposed algorithm and the implementation of the proposed algorithm in gradient descent optimization process. In Section three, the abilities of the proposed method to capture the exponential change in the belief variation of the cause when the belief in the effect is at its minimum is compared with the outputs from both the current method on a real casting data set. The paper is concluded in the final section along with short discussion on further research.

\section{THE Proposed AlgORITHM}

$$
\Delta w_{i j}^{(n)}=-\eta^{(n)} \frac{\partial E}{\partial w_{i j}^{(n)}}
$$

In this section, a novel approach for improving the training efficiency of back propagation neural network algorithms (BPM/AG) is proposed. The proposed algorithm modifies the initial search direction by changing the gain value adaptively for each node. Gain update expressions as well as weight and bias update expressions for output and hidden nodes have also been proposed. These expressions have been derived using same principles as used in deriving weight updating expressions.

$$
d^{(n)}=-\frac{\partial E}{\partial w_{i j}^{(n)}}\left(c_{j}^{(n)}\right)=g^{(n)}\left(c_{j}^{(n)}\right)
$$

The following iterative algorithm has been proposed for changing the gradient based search direction using a gain value.

Consider a multilayer feed-forward network, as used in standard back propagation algorithm [12]. Suppose that for a particular input pattern $o^{0}$, the desired output is the teacher pattern $t=\left[t_{1} \ldots t_{n}\right]^{T}$, and the actual output is $o_{k}^{L}$, where $L$ denotes the output layer. The error function on that pattern is defined as,

Let $o_{k}^{s}$ be the activation values for the $k^{\text {th }}$ node of layer $s$, and let $o^{s}=\left[o_{1}^{s} \ldots o_{n}^{s}\right]^{T}$ be the column vector of activation values in the layer $s$ and the input layer as layer 0 . Let $w_{i j}^{s}$ be the weight values for the connecting link between the $i^{\text {th }}$ node in layer $s-1$ and the $j^{\text {th }}$ node in layer $s$, and let $w_{j}^{s}=\left[w_{1 j}^{s} \ldots w_{n j}^{s}\right]^{T}$ be the column vector of weights from layer $s-1$ to the $j^{\text {th }}$ node of layer $s$. The net input to the $j^{\text {th }}$ node of layer $s$ is defined as $n e t_{j}^{s}=\left(w_{j}^{s}, o^{s-1}\right)=\sum_{k} w_{j, k}^{s} o_{k}^{s-1}, \quad$ and let $n e t^{s}=\left[n e t_{1}^{s} \ldots n e t_{n}^{s}\right]^{T}$ be the column vector of the net input values in layer $s$. The activation value for a node is given by a function of its net inputs and the gain parameter ${ }^{c_{j}}$; where $f$ is any function with bounded derivative.

Initialize the initial weight vector with random values and the vector of gain values with unit values. Repeat the following Steps 1 and 2 on an epoch-by-epoch basis until the given error minimization criteria are satisfied.

Step 1 By introducing gain value into activation function, calculate the gradient of error with respect to weights by using Equation (5), and gradient of error with respect to the gain parameter by using Equation (7).

Step 2 Use the gradient weight vector and gradient of gain vector calculated in Step 1 to calculate the new weight vector and vector of new gain values for use in the next epoch.

This information is now used to derive an expression for modifying gain values for the next epoch. Most of gradient based optimization methods use the following gradient descent rule:

where $\eta^{(n)}$ is the learning rate value at step $n$ and the gradient based search direction at step $\mathrm{n}$ is: $d^{(n)}=-\frac{\partial E}{\partial w_{i j}^{(n)}}=g^{(n)}$.

In the proposed algorithm the gradient based search direction is modified by including the variation of gain value to yield

The derivation of the procedure for calculating the gain value is based on the gradient descent algorithm. The error function as defined in Equation (1) is differentiated with respect to the weight value $w_{i j}^{s}$. The chain rule yields,

$$
\begin{array}{r}
\frac{\partial E}{\partial w_{i j}^{s}}=\frac{\partial E}{\partial n e t^{s+1}} \cdot \frac{\partial n e t^{s+1}}{\partial o_{j}^{s}} \cdot \frac{\partial o_{j}^{s}}{\partial n e t_{j}^{s}} \cdot \frac{\partial n e t_{j}^{s}}{\partial w_{i j}^{s}} \\
=\left[-\delta_{1}^{s+1} \ldots-\delta_{n}^{s+1}\right] \cdot\left[\begin{array}{l}
w_{1 j}^{s+1} \\
\vdots \\
w_{n j}^{s+1}
\end{array}\right] \cdot f^{\prime}\left(c_{j}^{s} n e t_{j}^{s}\right) c_{j}^{s} \cdot o_{j}^{s-1}
\end{array}
$$

where $\delta_{j}^{s}=-\frac{\partial E}{\partial n e t_{j}^{s}}$. In particular, the first three factors of 
Equation (5) indicate that the following equation holds:

$$
\delta_{1}^{s}=\left(\sum_{k} \delta_{k}^{s+1} w_{k, j}^{s+1}\right) f^{\prime}\left(c_{j}^{s} n e t_{j}^{s}\right) c_{j}^{s}
$$

It should be noted that, the iterative formula as described in Equation (6) to calculate $\delta_{1}^{s}$ is the same as used in the standard back propagation algorithms [12] except for the appearance of the gain value in the expression. The learning rule for calculating weight values as given in Equation (3) is derived by combining (5) and (6).

In this approach, the gradient of error with respect to the gain parameter can also be calculated by using the chain rule as previously described; it is easy to compute as:

$$
\frac{\partial E}{\partial c_{j}^{s}}=\left(\sum_{k} \delta_{k}^{s+1} w_{k, j}^{s+1}\right) f^{\prime}\left(c_{j}^{s} n e t_{j}^{s}\right) n e t_{j}^{s}
$$

Then the gradient descent rule for the gain value becomes,

$$
\Delta c_{j}^{s}=\eta \delta_{j}^{s} \frac{n e t_{j}^{s}}{c_{j}^{s}}
$$

At the end of every epoch the new gain value is updated using a simple gradient based method as given by the following formula,

$$
c_{j}^{\text {new }}=c_{j}^{\text {old }}+\Delta c_{j}^{s}
$$

In gradient descent method, the search direction at each step is given by the local negative gradient of the error function, and the step size is determined by a learning rate parameter. Suppose at step $n$ in gradient descent algorithm, the current weight vector is $w^{n}$, and a particular gradient based search direction is $d^{n}$. The weight vector at step $n+1$ is computed by the following expression:

$$
w^{(n+1)}=w^{n}+\eta^{n} d^{n}
$$

where, $\eta^{n}$ is the learning rate value at $\operatorname{step} n$.

By using the proposed algorithm namely as Back-propagation with Adaptive Gain Variation (BPM/AG), the gradient based search direction is calculated at each step by using Equation (4).

\section{RESULTS AND DiSCUSSIONS}

The abilities of the proposed algorithm to capture the exponential change in the belief variation of the cause when the belief in the effect is at its minimum is compared with the outputs from both the conventional back propagation algorithm on a real casting data set used by Ransing [1]. The data was collected from 'Kaye Preistigne'- a pressure die casting foundry. A total of 14 defects were identified and associated with 43 process, material or design parameters. The data was collected for similar components over a period of one year. A total of 60 representative examples were finalised.
A belief value in the occurrence of defects was calculated as corresponding to the belief values representing the occurrence and non-occurrence of associated process, design and material parameters as given by the experts in the foundry. Three defects known as 'Porosity', 'Mismakes' and 'Dimensional' are identified.

For the purpose of comparison, the graphical variation of belief surfaces learnt by the conventional neural network, Ransing [1] and the proposed method is showed only on two defects which are 'Porosity' and 'Mismakes'.

Fig. 1, Fig. 2 and Fig. 3 shows the variation in the belief values in the occurrence of "The position of gate" for belief values for defects "Porosity" and "Mismakes" using the proposed method, Ransing's method and the neural network method. It can be easily observed the proposed method has an ability to accurately model the exponential rise in the belief values than the other two techniques.

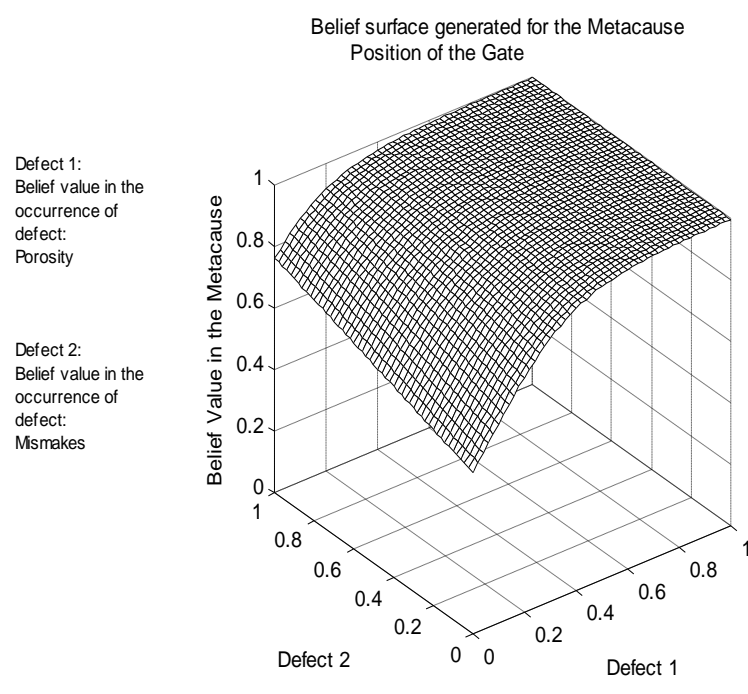

Fig. 1. 2D output surface for defects porosity and mismakes generated by the conventional neural network method.

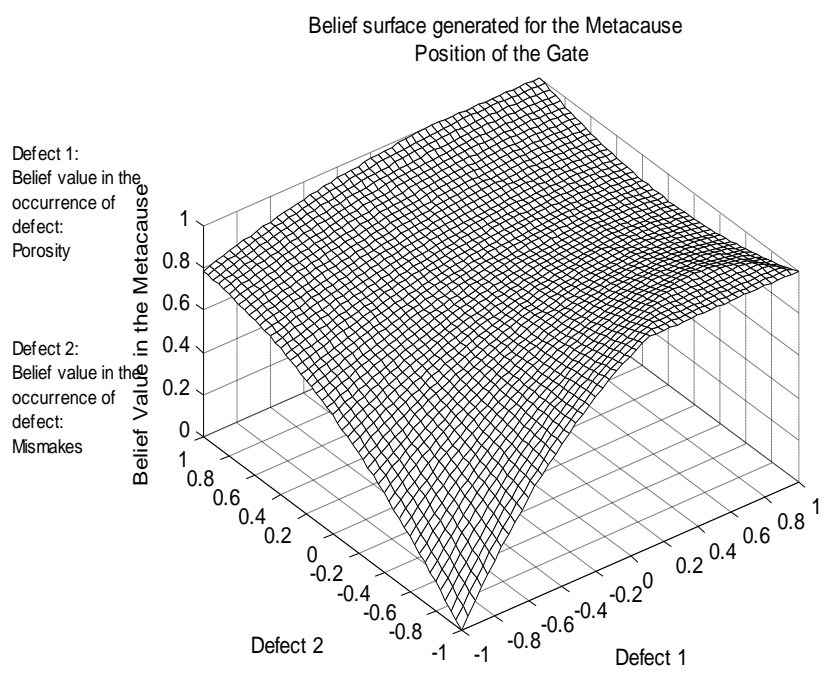

Fig. 2. 2D quadratic output surface for defects porosity and mismakes generated by ransing's method. 


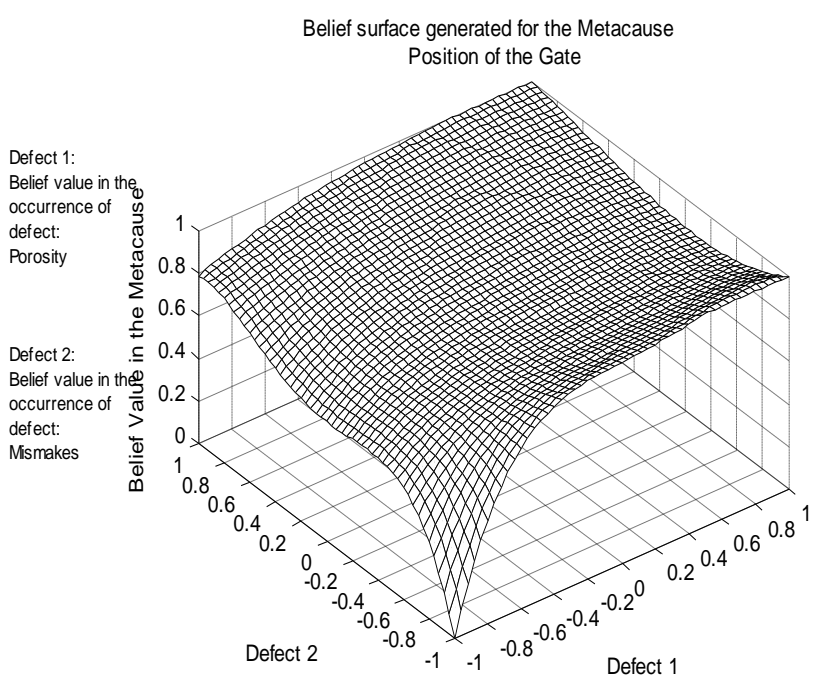

Fig. 3. 2D quadratic output surface for defects porosity and mismakes generated by the proposed method.

\section{CONCLUSION}

An enhancement to the conventional back propagation algorithm has been proposed in this paper. The method introduces mid points in the existing shape function formulation so that an exponential rise in the belief value variation can be modelled without introducing the effects of 'over-fitting'. The performance of the proposed method was compared with the method proposed by Ransing [1] and the conventional back propagation algorithm on the same casting data used by Ransing [1]. The results clearly demonstrated that the proposed method does not have limitations of the conventional back propagation techniques as identified by Ransing [1]. Furthermore, with the result of this research achievement, it will now be possible to correctly predict the sensitivity of process parameter variations to the occurrence of defects. This is an important area of research in a robust design methodology.

\section{ACKNOWLEDGMENT}

The authors would like to thank Universiti Tun Hussein Onn Malaysia (UTHM) for supporting this research.

\section{REFERENCES}

[1] M. R. Ransing, "Issues in learning cause and effect relationships from examples," With Particularly Emphasis on Casting Process, University of Wales Swansea, Swansea, 2002.

[2] B. Lally, L. T. Biegler, and H. Henein, "optimisation and continous casting. 1. problem formulation and solution strategy," Metallurgical Transactions B - Process Metallugy, vol. 22, pp. 641-648, 1991.

[3] J. Grum and J. M. Slabe, "The use of factorial design and response surface methodology for fast determination of optimal heat treatment conditions of different Ni-Co-Mo surfaced layers," Journal of Materials Processing Technology, vol. 155-156, pp. 2026-2032, 2004.

[4] A. Bendall, "Introduction to Taguchi methodology," Proceedings of the 1988 European Conference, pp. 1-14, 1988.

[5] R. N. Kackar, "Off-line quality control, parameter design and the Taguchi method,” Journal of Quality Technology, vol. 17, pp. 176-88, 1985.
[6] H. Singh and P. Kumar, "Optimizing cutting force for turned parts using Taguchi’s parameter design approach," Indian J. Eng. Mater. Sci., vol. 12, pp. 97-103, 2005.

[7] M. Perzyk and A. Kochanski, "Detection of cause of casting defetcs assisted by artificial neural networks: Proceedings of the institution of mechanical engineers, part B," Journal of Engineering Manufacture, vol. 217, pp. 1279-1284, 2003.

[8] R. L. Wilson and R. Sharda, "Bankruptcy prediction using neural networks,” Decision Support Systems, vol. 11, pp. 545-557, 1994.

[9] K. Funahashi, "On the approximate realization of continuous mappings by neural networks," Neural Networks, vol. 2, pp. 183-192, 1989.

[10] N. M. Nawi, M. R. Ransing, and R. S. Ransing, "Improving the gradient based search direction to enhance training efficiency of back propagation based neural network algorithms," Proceedings of the 26th International Conference of Innovative Techniques and Applications of Artificial Intelligent (SGAI06), pp. 45-48, 2006.

[11] C. M. Bishop, "Neural networks for pattern recognition," Oxford University Press, 1995

[12] D. E. Rumelhart, G. E. Hinton, and R. J. Williams, "Learning internal representations by error propagation," in D. E. Rumelhart and J. L. McClelland (eds), Parallel Distributed Processing. vol. 1, pp. 318-362. , 1986

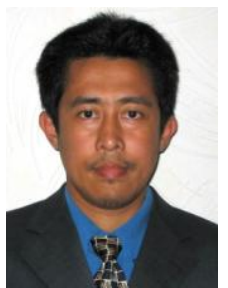

Nazri Mohd Nawi received his Bachelor degree in Computer Science from University of Science Malaysia (USM), Penang, Malaysia. His M.Sc. degree in computer science was received from University of Technology Malaysia (UTM), Skudai, Johor, Malaysia. He received his Ph.D. degree in Data Mining from Mechanical Engineering department, Swansea University, Wales United Kingdom. He had published over 20 papers in refereed journals and a technical committee for many symposiums, workshop, and conferences on this topic. $\mathrm{He}$ is currently an Associate Professor in Software Engineering Department at Universiti Tun Hussein Onn Malaysia (UTHM). His research interests are in optimization, data mining techniques and artificial neural networks.

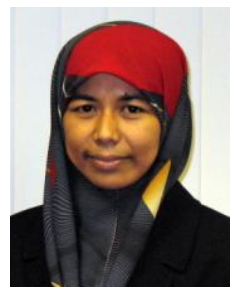

Zawati Harun received her Bachelor and Master degree in Material Engineering from University of Science Malaysia (USM), Penang,, Malaysia. She received her Ph.D. degree in Mechanical Engineering from Swansea University, Wales United Kingdom. She had published over 10 papers in refereed journals and a technical committee for many symposiums, workshop, and conferences on this topic. She is currently an Associate Professor in Material Engineering and Design Department at Universiti Tun Hussein Onn Malaysia (UTHM). Her research interests are in modeling and simulation, porous material, drying and membrane.

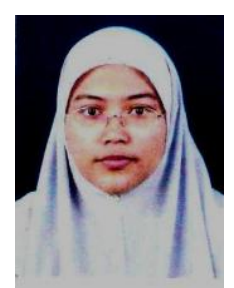

Noorhnaiza Wahid received her B. Sc (Hons.) degree in computer science and M.Sc degree in computer science (multimedia system) from University Putra Malaysia in 1997 and 2000 respectively. She obtained her degree in $\mathrm{PhD}$ from University of Sydney, Australia in 2011. She has been with the University Tun Hussein Onn Malaysia as lecturer since 2001. She is currently a senior lecturer with a Department of Multimedia, Faculty of Computer Science and Information Technology, in Universiti Tun Hussein Onn Malaysia. Her current research interest include data mining which involve bio-inspired algorithms (mainly, swarm-based optimization algorithms) and multimedia system. 